\title{
Video Article \\ A Model of Self-limited Acute Lung Injury by Unilateral Intra-bronchial Acid Instillation
}

\author{
Alexander H. Tavares ${ }^{1}$, Jennifer K. Colby ${ }^{1}$, Bruce D. Levy ${ }^{1}$, Raja-Elie Edward Abdulnour ${ }^{1}$ \\ ${ }^{1}$ Pulmonary and Critical Care Medicine, Department of Internal Medicine, Brigham and Women's Hospital, Harvard Medical School
}

Correspondence to: Raja-Elie Edward Abdulnour at rabdulnour@bwh.harvard.edu

URL: https://www.jove.com/video/60024

DOI: doi:10.3791/60024

Keywords: Immunology and Infection, Issue 150, acid aspiration, acute lung injury, acute respiratory distress syndrome (ARDS), selective intrabronchial instillation, unilateral, resolution

Date Published: 8/30/2019

Citation: Tavares, A.H., Colby, J.K., Levy, B.D., Abdulnour, R.E. A Model of Self-limited Acute Lung Injury by Unilateral Intra-bronchial Acid Instillation. J. Vis. Exp. (150), e60024, doi:10.3791/60024 (2019).

\section{Abstract}

Selective intra-bronchial instillation of hydrochloric acid $(\mathrm{HCl})$ to the murine left mainstem bronchus causes acute tissue injury with histopathologic findings similar to human acute respiratory distress syndrome (ARDS). The resulting alveolar edema, alveolar-capillary barrier damage, and leukocyte infiltration predominantly affect the left lung, preserving the right lung as an uninjured control and allowing animals to survive. This model of self-limited acute lung injury enables investigation of tissue resolution mechanisms, such as macrophage efferocytosis of apoptotic neutrophils and restitution of alveolar-capillary barrier integrity. This model has helped identify important roles for resolution agonists, including specialized pro-resolving mediators (SPMs), providing a foundation for the development of new therapeutic approaches for patients with ARDS.

\section{Video Link}

The video component of this article can be found at https://www.jove.com/video/60024/

\section{Introduction}

Acute respiratory distress syndrome (ARDS) is an important cause of acute respiratory failure ${ }^{1}$. It is a common and lethal or disabling disease that occurs in $10 \%$ of all patients admitted to intensive care units worldwide ${ }^{2}$. According to the Berlin definition ${ }^{3}$, ARDS is defined by the acute onset of hypoxemic respiratory failure ( $<1$ week) and bilateral pulmonary infiltrates on chest radiographs that are not explained by cardiac failure ${ }^{4}$. The underlying pathobiology is characterized by an excessive inflammatory response. The lung can be injured directly, such as in pneumonia or with gastric acid aspiration, or indirectly, such as in sepsis or after multiple blood transfusions ${ }^{4}$. Following the initial insult, ARDS pathogenesis progresses in three phases: exudative, proliferative, and fibrotic phases ${ }^{1}$. These phases are characterized by distinct molecular and cellular immune and repair mechanisms that determine the prognosis for ARDS patients. Supportive care remains the mainstay for ARDS patients; currently, there are no effective pharmacological treatments for ARDS, so there is an urgent need for new research on this devastating condition ${ }^{4}$.

Dysregulation of the innate immune response during the exudative phase contributes to the acute onset of ARDS and associated respiratory failure ${ }^{1}$. Potent pro-inflammatory mediator signaling orchestrates the initial immune responses, leading to disruption of the alveolar-capillary barrier, diffuse alveolar edema, and neutrophil infiltration to the site of lung tissue injury ${ }^{4}$. In ARDS, ineffective braking signals for acute inflammation predispose to lung failure and can delay timely catabasis of the injured lung tissue ${ }^{5}$. To that end, preclinical investigation into the endogenous initiating and pro-resolution mechanisms of ARDS may uncover novel therapeutic strategies. Such investigation requires selflimited experimental in vivo models of acute lung injury that closely resemble features of human ARDS, permitting interrogation of mechanisms underlying the initiation and resolution phases of tissue injury.

The murine model presented here produces direct acute lung injury that demonstrates the cardinal pathobiological processes of exudative ARDS, namely alveolar-capillary barrier disruption and neutrophil infiltration. The method relies on selective intra-bronchial instillation of $\mathrm{HCl}$ through cannulation of the left mainstem bronchus, localizing the injury and inflammatory response to the left lung; the uninjured right lung can be used as an internal control for select determinations of tissue injury and inflammation. In addition, unilateral lung injury is non-lethal and unveils a resolution program. This offers a distinct window into resolution of lung inflammation that can be leveraged for identification of endogenous pro-resolving mediators and cellular mechanisms and to open new therapeutic avenues for ARDS that emphasizes resolution physiology and pharmacology.

\section{Protocol}

All animal procedures below have been reviewed and approved by the Institutional Animal Care And Use Committee at Brigham and Women's Hospital (Protocol \#2016N000356). 
NOTE: Sterile technique was followed for all survival procedures. A sterile field was established for each surgery using a sterile drape towel, while surgeons wore sterile surgical gloves, caps, masks, and clean laboratory coats. All surgical instruments were sterilized using an autoclave, and sterility was maintained using a bead sterilizer.

\section{Preparation of $0.1 \mathrm{~N} \mathrm{HCl}$}

1. Add $11 \mathrm{~mL}$ of $\mathrm{ddH}_{2} \mathrm{O}$ to an amber glass bottle. Slowly add $1 \mathrm{~mL}$ of $37 \% \mathrm{HCl}(12 \mathrm{~N})$ to create a $1 \mathrm{~N} \mathrm{HCl}$ working stock CAUTION: Make sure $\mathrm{HCl}$ is added into the water. This is a safety concern because adding water directly to the acid can cause acid to boil and splash out of the bottle. When handling concentrated $\mathrm{HCl}$, make sure the acid is kept in a vented chemical hood and the appropriate personal protective equipment is worn, including lab coat, gloves, and safety glasses.

2. Slowly add $4 \mathrm{~mL}$ of the previously diluted $\mathrm{HCl}$ working stock into $35 \mathrm{~mL}$ of $\mathrm{ddH}_{2} \mathrm{O}$ in a $50 \mathrm{~mL}$ conical tube to create a $0.1 \mathrm{~N} \mathrm{HCl}$ experimental stock.

3. Measure the $\mathrm{pH}$ of the experimental stock using an electronic $\mathrm{pH}$ probe after two-point calibration using low $\mathrm{pH}$ solutions. Titrate to $\mathrm{pH}$ 1.1 using $\mathrm{NaOH}$ or $\mathrm{HCl}$ stock solutions as needed so that the final volume is $40 \mathrm{~mL}$.

NOTE: Measuring the low $\mathrm{pH}$ values can be difficult. To ensure accurate measurement, make sure the pH probe is properly calibrated using low $\mathrm{pH}$ standards to avoid over-extrapolation of the measurement.

4. Immediately before the experiment, filter $1-2 \mathrm{~mL}$ of the experimental $\mathrm{HCl}$ stock through a $0.22 \mu \mathrm{m}$ sterile filter into a sterile microcentrifuge tube.

\section{Selective Intra-bronchial Instillation of $\mathrm{HCl}$}

\section{Preparing the surgical area}

1. Induce general anesthesia by delivering a ketamine $(100 \mathrm{mg} / \mathrm{kg})$ and xylazine $(10 \mathrm{mg} / \mathrm{kg})$ mixture by intraperitoneal injection. Ensure the mouse is completely anesthetized by gently squeezing the tip of the tail or hind foot. Lack of withdrawal response is required prior to making a skin incision. Administer additional anesthesia boluses, if needed.

2. Deliver $0.1 \mathrm{mg} / \mathrm{kg}$ of buprenorphine subcutaneously under the scruff of the neck. Pre-operative analgesic will strengthen the effect of the anesthesia and will ameliorate pre- and post-operative pain resulting from the procedure.

3. Use electric clippers to gently shave the surgical area on the ventral surface of the mouse, below the chin in the cervical region of the throat, using slow downward strokes. Remove loose fur to fully expose the underlying skin.

4. Prepare the surgical area by swabbing the shaved site with $10 \%$ povidone-iodine solution. After applying the aseptic solution, clean the site using a $70 \%$ isopropyl alcohol swab. Repeat this step $3 x$.

\section{Isolating the trachea}

1. Place the mouse in a supine position on a clean surgical board and cover the mouse in a sterile surgical drape while maintaining exposure of the surgical area. Secure the drape in place.

2. Make a $0.5 \mathrm{~cm}$ longitudinal incision in the skin above the trachea and salivary glands. Use slightly curved serrated forceps to carefully pull back the skin and gently separate the salivary glands to expose the tracheal muscles.

3. Using serrated forceps for blunt dissection, gently push apart the paratracheal muscles and tease away the fascia that is surrounding the trachea until the cartilaginous rings of the trachea are completely exposed.

4. Use fully curved serrated forceps to lift the trachea and separate the connective tissue between the retro-trachea and the retro-fascia. Once the connective tissue is detached, the tip of the forceps should slide completely behind the trachea.

5. Keep the curved forceps behind the trachea and grasp a $10-15 \mathrm{~cm}$ piece of $4-0$ braided silk suture with the tips of the forceps. Pull the suture behind the trachea so that there is an even length on either side.

6. Once the suture is in place, gently pull the sides of the suture towards the posterior of the mouse and hold the sides in place.

3. Selectively cannulating the left mainstem bronchus and instilling $\mathrm{HCl}$

1. Take a $24 \mathrm{G} \mathrm{x} \mathrm{3/4"} \mathrm{angiocatheter} \mathrm{and} \mathrm{insert} \mathrm{the} \mathrm{needle,} \mathrm{bevel} \mathrm{up,} \mathrm{into} \mathrm{the} \mathrm{anterior} \mathrm{region} \mathrm{of} \mathrm{the} \mathrm{trachea} \mathrm{between} \mathrm{the} \mathrm{first} \mathrm{and} \mathrm{second}$ tracheal rings. Once proper insertion is confirmed by direct visualization of the needle tip in the tracheal lumen, release the suture and advance the cannula over the needle and into the trachea until resistance is reached, then withdraw the needle. Angle the direction of insertion towards the left main stem bronchus for selective instillation into the left lung

2. Once the cannula is in place, firmly grasp the injection port to prevent the catheter from shifting.

3. Using a P200 pipette and sterile P200 pipette tips, instill $2.5 \mathrm{~mL} / \mathrm{kg}$ (50 $\mu \mathrm{L}$ for a $20 \mathrm{~g}$ mouse) of sterile filtered $0.1 \mathrm{~N} \mathrm{HCl}$ into the catheter, followed by an identical volume of air.

4. Rapidly withdraw the catheter and lift the surgical board to a $60^{\circ}$ angle for $30 \mathrm{~s}$.

4. Closing the surgical area

1. Lay the surgical board flat and remove the suture from behind the trachea.

2. Use 4-0 wax coated braided silk suture to close the skin incision using 2-3 stitches.

\section{Post-operative Care}

1. Once the incision is closed, place the mouse on its left side on a warm heating pad until the mouse recovers from anesthesia. Begin monitoring the mouse for pain and activity level before returning it to normal housing.

NOTE: Buprenorphine should be administered at $0.1 \mathrm{mg} / \mathrm{kg}$ subcutaneously every $6-12 \mathrm{~h}$ for the first $24 \mathrm{~h}$. If persistent breakthrough pain is present, extend the analgesic regimen until the pain subsides. 


\section{Whole Lung Bronchoalveolar Lavage (BAL) and Leukocyte Immunophenotyping}

1. Euthanize the mouse by administering $3 x$ the dose of ketamine/xylazine used in step 2.1.1.

1. To differentiate interstitial and intravascular neutrophils, intravenously inject a selected fluorophore-labeled Ly6G antibody $\mathbf{5}$ min prior to euthanasia. This label should be suitable for detection by flow cytometry to distinguish intravascular neutrophils from lung interstitial and alveolar neutrophils, which will be labeled during the tissue preparation with a different fluorophore (see below).

2. Place the mouse on a surgical board and hook the anterior incisors around a loop of 2-0 braided silk suture.

3. Follow steps 2.2.2-2.2.6. to prepare the trachea for cannulation. NOTE: Make sure the diaphragm is not punctured to maximize trans-alveolar inflating pressure during lung lavage; left lung compliance decreases following injury, which may require a higher trans-alveolar pressure requirement for lung lavage.

4. Cannulate the trachea following step 2.3.1, but do not advance the catheter below the carina; insert the catheter parallel to the trachea.

5. With the catheter inserted, tie the suture around the trachea to hold the catheter in place.

6. Instill and withdraw two consecutive $1 \mathrm{~mL}$ aliquots of ice-cold PBS -/- (without magnesium or calcium) with $0.6 \mathrm{mM}$ EDTA using a $1 \mathrm{cc}$ syringe. For immunophenotyping by flow cytometry, remove each aliquot and return to a $5 \mathrm{~mL}$ polystyrene FACS tube on ice.

7. To ensure euthanasia, perform a thoracotomy using surgical scissors followed by cardiac puncture. The lungs may be harvested for further processing.

8. Centrifuge the BAL for $10 \mathrm{~min}$ at $800 \mathrm{~g}$ at $4{ }^{\circ} \mathrm{C}$ to pellet the cells.

9. Decant the supernatant into a $2 \mathrm{~mL}$ microcentrifuge tube and aliquot into $1.5 \mathrm{~mL}$ microcentrifuge tubes. Store at $-80^{\circ} \mathrm{C}$ for subsequent analysis.

10. Resuspend the cell pellet in PBS -/- with $2 \%$ FBS for leukocyte differential analysis by flow cytometry.

11. To differentiate interstitial and intravascular neutrophils, remove the left and right lung separately and process the lungs for flow cytometry as in Abdulnour et al. 20146.

12. Stain the resulting cell suspension using selected FACS antibodies, making sure to stain for Ly6G that is conjugated with a different fluorophore than the Ly6G antibody from step 4.1.1.

\section{Assessment of Alveolar Barrier Permeability Using Evan's Blue Dye (EBD)}

1. Intravenously inject Evan's Blue Dye $(40 \mathrm{mg} / \mathrm{kg}) 30 \mathrm{~min}$ prior to euthanasia.

2. Euthanize the mouse using ketamine/xylazine overdose (step 4.1).

3. To measure alveolar barrier integrity, follow steps 4.2-4.9 for BAL collection.

4. Transfer $100 \mu \mathrm{L}$ of BALF to a clear bottom 96 -well microplate, along with $100 \mu \mathrm{L}$ of duplicate EBD standards. Use PBS -/- as a blank.

5. Use a microplate reader to measure the absorbance of the BALF at $620 \mathrm{~nm}$ and $740 \mathrm{~nm}$. Use the absorbance at $740 \mathrm{~nm}$ to correct for heme contamination in the samples ${ }^{7}$.

6. To measure vascular barrier integrity, perfuse the lungs by slowly injecting $5 \mathrm{~mL}$ of ice-cold PBS -/- through the right ventricle of the heart. Remove the left lung.

7. Dry the left lung for $72 \mathrm{~h}$ at $58{ }^{\circ} \mathrm{C}$ to remove excess water.

8. Process the dried lung tissue as in Radu and Chernoff $2013^{8}$, and measure the absorbances at $620 \mathrm{~nm}$ and $740 \mathrm{~nm}$.

\section{Lung Histology}

1. Cannulate the trachea by following steps 4.1-4.5.

2. To pressure fix the lungs at $20 \mathrm{~cm} \mathrm{H}_{2} \mathrm{O}$, use a ring stand and clamp to elevate a $60 \mathrm{~mL}$ syringe fitted with valve-controlled tubing and filled with a select fixative solution (e.g., zinc fixative) so that the meniscus of the fixative solution is $20 \mathrm{~cm}$ above the lungs.

3. Attach the tubing to the catheter and open the value. Slowly fill the lungs with fixative until they stop inflating.

4. Remove the catheter $3 / 4$ of the way out of the trachea. Tie off the trachea with suture before fully removing the catheter to minimize loss of fixative.

5. Remove the lungs and heart en bloc.

NOTE: Make sure the lungs are not punctured during the removal to retain the pressure infused fixative.

6. Fix the lungs for $24 \mathrm{~h}$ in $25 \mathrm{~mL}$ of fixative at room temperature.

7. Wash the fixed lungs for sequential 20 min intervals in PBS $-/-, 30 \%$ ethanol, and $50 \%$ ethanol.

8. After the last wash, store the lungs in $70 \%$ ethanol for histology processing as in Eickmeier et al. $2013^{9}$.

\section{Representative Results}

Selective intra-bronchial $\mathrm{HCl}$ instillation results in unilateral acute lung injury

The method of selective intra-bronchial instillation of $\mathrm{HCl}$ into the left mainstem bronchus is illustrated in Figure 1A. The consequent acute lung injury involves the entire left lung, and following intravenous administration of EBD and lung perfusion, the EBD remained only in the left lung (Figure 1B). EBD extravasation into the left lung was quantified and found to be significantly increased relative to sham selective instillation (Figure 1C; adapted from Abdulnour et al. $2014^{6}$ ). In response to lung injury, circulating leukocytes diapedese into the inflamed tissue. In this model, vascular neutrophils undergo trans-endothelial migration into the injured lung interstitium. Interstitial neutrophils accumulated in the left lung $24 \mathrm{~h}$ after $\mathrm{HCl}$ instillation, in contrast to the right lung where few interstitial neutrophils are observed (Figure 1D). These results indicate that the selective left mainstem intra-bronchial instillation method resulted in murine acute lung injury that was largely localized to the left lung and produced pathological changes that are also seen with human ARDS, including increased alveolar-capillary barrier breach and neutrophil infiltration. 


\section{Unilateral acute lung injury enables investigation of resolution mechanisms}

To study the resolution phase of acid-induced acute lung injury mice must be able to survive the initial insult. Distinct from intratracheal $\mathrm{HCl}$, instillation into only the left mainstem bronchus leads to a self-limited injury with uniform survival in otherwise healthy mice. Lungs can be obtained from mice at either early or later timepoints as in Figure 2A. Lung histology shows tissue injury and inflammation at the organ and cellular level with exudative inflammation $24 \mathrm{~h}$ after injury characterized by marked alveolar edema and neutrophil infiltration in the left lung. Note that there is no significant injury or leukocyte influx into the uninjured control right lung (Figure 2A). $72 \mathrm{~h}$ after injury, edema and cellular infiltrates are substantially decreased, representing a resolving exudative phase. Alveolar neutrophils can be monitored by flow cytometry $\left(C D 45^{+} /\right.$ $\mathrm{CD}^{-} / \mathrm{F} 4 / 80^{-} / \mathrm{Ly} 6 \mathrm{G}^{+} / \mathrm{CD} 11 \mathrm{~b}^{+}$) obtained by whole lung lavage. Neutrophils increase in the left lung $24 \mathrm{~h}$ following the initial injury and decrease substantially at 48 and $72 \mathrm{~h}$ (Figure 2B). If later time points are investigated, the neutrophil numbers will return to baseline and mechanisms in later phases of catabasis, such as fibroproliferative responses, can be studied.

A

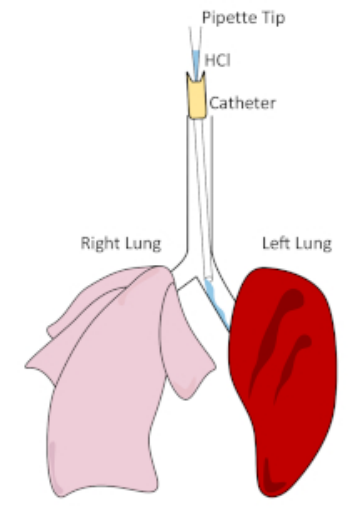

B

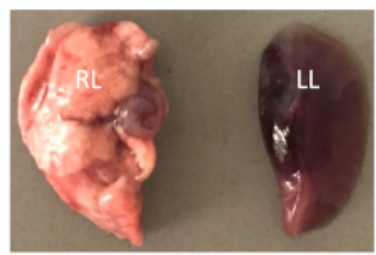

C

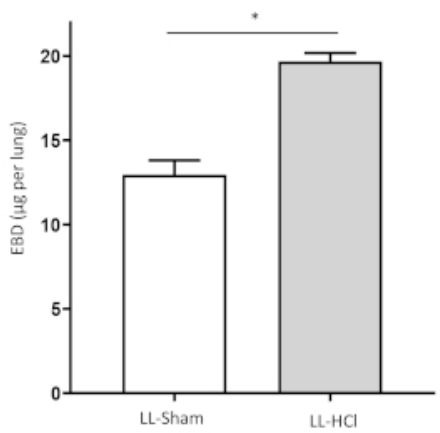

D

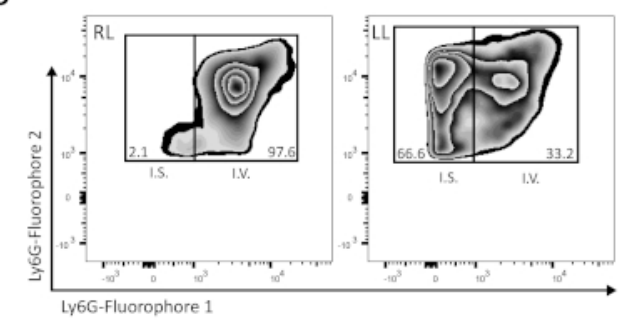

Figure 1: Selective intra-bronchial $\mathrm{HCl}$ instillation produces unilateral lung injury defined by alveolar barrier breach and neutrophil infiltration. (A) Representation of the cannulation of the murine left mainstem bronchus for selective instillation of $\mathrm{HCl}$ into the left lung. (B) Resected right (RL) and left (LL) lungs exposed to selective acid instillation and perfused following intravenous Evan's blue dye. (C) Quantification of interstitial Evan's blue dye from homogenized, perfused lung $24 \mathrm{~h}$ after acid injury or sham control; figure adapted from Abdulnour et al. $2014^{6}$. Values represent mean \pm SEM, where $n \geq 5$. ${ }^{*} p<0.05$, Mann-Whitney $U$ Test. (D) Representative flow cytometry of intravascular (I.V.; fluorophore 1) and interstitial (I.S.; fluorophore 2) neutrophils as percent of total CD $45^{+}$cells in processed lung $24 \mathrm{~h}$ after acid injury. Please click here to view a larger version of this figure. 
A
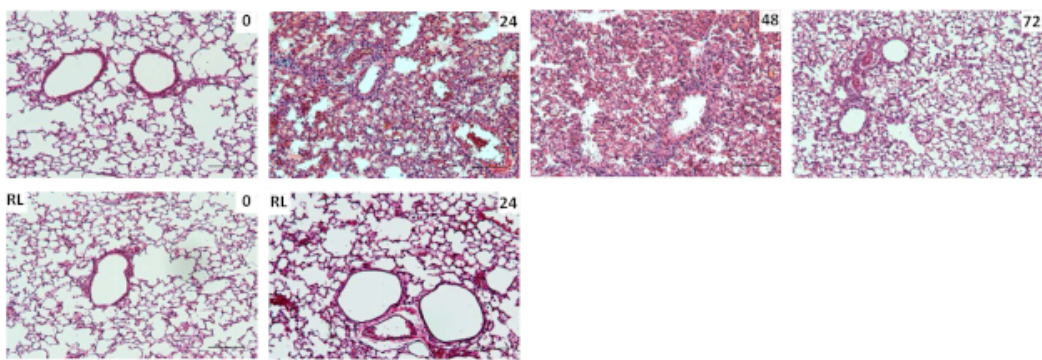

B

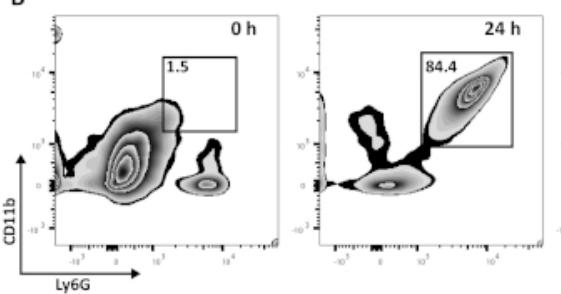

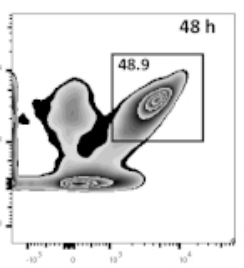

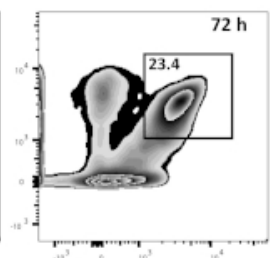

Figure 2: Unilateral acute lung injury is self-resolving. (A) Representative H\&E histology (10x) of left lungs obtained from naïve mice (0 h) or mice 24, 48, $72 \mathrm{~h}$ after injury, along with the associated right lung from the same mouse (Scale bar $=250 \mu \mathrm{m})$. (B) Representative flow cytometry of alveolar neutrophils $\left(\mathrm{Ly}_{6 \mathrm{G}}{ }^{+} \mathrm{CD} 11 \mathrm{~b}^{+}\right.$) obtained from whole lung lavage as percent of total CD $45^{+}$cells in naïve $(0 \mathrm{~h})$ mice or mice 24,48, and $72 \mathrm{~h}$ after acid injury. Please click here to view a larger version of this figure.

\section{Discussion}

The intra-bronchial instillation method described here uses selective cannulation of the left mainstem bronchus to instill $\mathrm{HCl}$ into the left lung, resulting in unilateral and self-limited murine acute lung injury. This murine acid lung injury model closely represents the inflammatory response, histopathology, and physiological dysfunction seen in human ARDS, where gastric acid aspiration is a common precipitant or contributing factor $^{4}$. Exposure of the murine airway to low $\mathrm{pH} \mathrm{HCl}$ results in increased permeability of the alveolar-capillary barrier, alveolar edema, and profound neutrophil infiltration at the site of injury. These events are not observed in the uninjured right lung. In addition, this model produces rapid inflammatory responses that peak within $24 \mathrm{~h}$ following acid instillation, and shares changes in gene expression with human ARDS, such as the differential expression of phospholipase $D$ isoforms ${ }^{10}$.

Although this murine preclinical model reproduces many of the features of ARDS at the molecular, cellular, and tissue levels, it does not fully recapitulate human ARDS. The definition of ARDS includes bilateral lung involvement ${ }^{3}$, whereas the instillation method described here results by design in unilateral lung disease. Moreover, the animals do not require continuous mechanical ventilation, immobility, or parenteral sedation. Results presented here (vide supra) and elsewhere ${ }^{6,9,11,12,13}$ demonstrate that unilateral acid-induced lung injury reproduces most of the pathological hallmarks of ARDS while providing the unique opportunity to use the right lung as internal control and to study the resolution phase of this disease. As such, the model discussed here models ARDS pathobiology, but also enables mechanistic investigation of fundamental lung tissue responses to injury and resolution mechanisms that may be relevant for addressing this important disease.

Instillation of $\mathrm{HCl}$ represents direct acute lung injury, so it is modeling aspects of the pathophysiology associated with aspiration pneumonitis. In addition, the initial left lung insult in this model is generated using sterile $\mathrm{HCl}$ rather than bacteria-laden gastric contents seen in some human aspiration events that can also lead to pneumonia ${ }^{14}$. In humans, aspiration of pathogenic bacteria can result in secondary bacterial pneumonia that exacerbates the acute inflammatory response, prolonging the initial lung injury and increasing patient susceptibility to develop ARDS ${ }^{14}$. This potential limitation has been addressed by investigators purposely instilling pathogenic bacteria Escherichia coli $(E \text {. coli })^{15}$ after sterile $\mathrm{HCl}$. Additionally, this method has been used to investigate pathogen-mediated inflammation; unilateral bacterial pneumonia can be induced by selective left lung instillation of bacteria, such as $E$. coli ${ }^{16,17}$, Pseudomonas aeruginosa ${ }^{16}$, and Streptococcus pneumoniae ${ }^{18}$. The self-limited acute lung injury model described here may also be used to study ventilator-induced lung injury (VILI), an important cause of increased mortality in human ARDS ${ }^{19}$. Experimental animal models of VILI usually involve mechanical ventilation in naïve mice with tidal volumes that are much higher than what is clinically used to cause lung injury $\left(>15 \mathrm{~mL} / \mathrm{kg}\right.$; see previous work $\left.{ }^{20,21}\right)$. Towards a more clinically relevant model of VILI, intra-bronchial acid instillation as described here may be used first to induce non-lethal lung injury followed by mechanical ventilation at tidal volumes within clinical range $(6-12 \mathrm{~mL} / \mathrm{kg})$. This hypothetical animal model may allow investigators to study VILI in a clinically relevant manner once developed and validated. Together, these murine models highlight the versatility of the selective intrabronchial instillation method to generate unilateral lung insults that closely resemble pathologies associated with human lung diseases.

In addition to allowing selective instillation of various noxious agents to the left lung, the technique of intra-bronchial instillation after tracheostomy does not require extended training, long procedure time, or complex equipment, and in experienced hands causes minimal distress to the animals. Despite this, several issues may occur during the selective $\mathrm{HCl}$ instillation procedure that can impact experimental results. Improper cannulation of the left main stem bronchus can result in bilateral lung injury that decreases survival of experimental mice and confounds the use of the right lung as an uninjured internal control. This can be avoided by angling the catheter sufficiently towards the left lung during cannulation until resistance is reached. After the injection of $\mathrm{HCl}$, a bolus of air should be injected, the catheter rapidly removed, and the 
surgical board brought upright to a $60^{\circ}$ angle. These steps are crucial for ensuring that the acid reaches the distal airways of the left lung and prevents the reflux of acid into the right lung and trachea, which may cause proximal injury. Within $24 \mathrm{~h}$ following instillation, the injury in the left lung is diffuse with extensive pulmonary edema, affecting both the distal and proximal left lung.

During method development in adult $8-12$ week old mice, $2.5 \mathrm{~mL} / \mathrm{kg}$ of intra-bronchial $\mathrm{HCl}$ produced substantial yet sublethal acute lung injury; lower doses of $\mathrm{HCl}$ did not result in reproducible and homogenous lung injury. Although we have not performed this model in younger (e.g., 3-6 weeks old) or older mice (e.g., 10-14 months old), we anticipate that weight-based dosing of $\mathrm{HCl}$ will result in a lung injury phenotype similar to what is noted in 8-12 week old mice. We recommend that investigators titrate $\mathrm{HCl}$ doses to achieve the desired degree of lung injury prior to performing experiments with mice at extremes of weight.

This selective acid instillation procedure offers a non-lethal murine model of sterile tissue inflammation that reduces the need for supportive care, such as mechanical ventilation. With extended survival of injured mice, the acid-induced inflammation has enough time to self-resolve. The resolution phase of this model has been used to identify temporally regulated endogenous bioactive lipid mediators, termed specialized pro-resolving mediators (SPMs), such as lipoxin $\mathrm{A}_{4}\left(\mathrm{LXA}_{4}\right)$, maresin 1 (MaR1), and resolvins ${ }^{6,11,12,16}$. Administering exogenous SPMs to injured mice quickens the resolution of acid induced lung injury by dampening inflammatory mechanisms and promoting catabasis of the injured lung tissue. These SPMs promote the clearance of alveolar edema ${ }^{12}$, increase the efferocytosis of apoptotic neutrophils by recruited macrophages ${ }^{16}$ and accelerate the re-epithelialization of the airways and alveoli ${ }^{12}$ to reduce vascular leakage and tissue hypoxia. In a model of pathogeninduced lung injury, 15-epi-resolvin D1 also exhibited antimicrobial actions through increased bacterial phagocytosis by macrophages and enhanced bacterial clearance from the infected lung ${ }^{16}$. Investigating these endogenous resolution mechanisms provides insight into potential novel therapeutic strategies for patients with ARDS 5 .

To best study the spatiotemporal regulation of resolution mechanisms, in vivo experimental models are needed. Acute lung injury models must include relevant acute inflammatory responses and organ dysfunction with engagement of host resolution promoting molecular and cellular processes. These mechanisms can be quantified using established resolution indices ${ }^{22}$. The selective intra-bronchial instillation method to generate unilateral acute lung injury has proven useful in this regard to probe endogenous resolution mediators and pathways. Future studies that deepen our understanding of these active resolution processes have the promise of leading to therapeutic agonists that mimic the bioactions of endogenous lipid mediators to enhance the resolution of inflammation and mitigating the morbidity and mortality of ARDS and other important lung diseases.

\section{Disclosures}

The authors have nothing to disclose.

\section{Acknowledgments}

The authors would like to thank Dr. Joseph Mizgerd for his contributions to the development of the selective intra-bronchial method and for his helpful comments and review of the manuscript. This work was supported by National Institutes of Health grants P01GM095467 (B.D.L.) and K08HL130540 (R.E.A.).

\section{References}

1. Baron, R. M., Levy, B. D. Acute Respiratory Distress Syndrome. In Harrison's Principles of Internal Medicine, 20e. (eds. Jameson, J. L. et al.). McGraw-Hill Education (2018).

2. Bellani, G. et al. Epidemiology, Patterns of Care, and Mortality for Patients With Acute Respiratory Distress Syndrome in Intensive Care Units in 50 Countries. The Journal of the American Medical Association. 315 (8), 788-800 (2016).

3. The ARDS Definition Task. Acute Respiratory Distress Syndrome: The Berlin DefinitionThe Berlin Definition of ARDS. The Journal of the American Medical Association. 307 (23), 2526-2533 (2012).

4. Thompson, B. T., Chambers, R. C., Liu, K. D. Acute Respiratory Distress Syndrome. New England Journal of Medicine. 377 (6), $562-572$ (2017).

5. Krishnamoorthy, N., Levy, B. D., Walker, K. H., Abdulnour, R.-E. E., Engstrom, B. D. Specialized Proresolving Mediators in Innate and Adaptive Immune Responses in Airway Diseases. Physiological Reviews. 98 (3), 1335-1370 (2018).

6. Abdulnour, R.-E. E. et al. Maresin 1 biosynthesis during platelet-neutrophil interactions is organ-protective. Proceedings of the National Academy of Sciences of the United States of America. 111 (46), 16526 LP-16531 (2014).

7. Chen, H. et al. Pulmonary permeability assessed by fluorescent-labeled dextran instilled intranasally into mice with LPS-induced acute lung injury. PLOS ONE. 9 (7), (2014).

8. Radu, M., Chernoff, J. An in vivo assay to test blood vessel permeability. Journal of Visualized Experiments. (73), e50062 (2013).

9. Eickmeier, O. et al. Aspirin-triggered resolvin D1 reduces mucosal inflammation and promotes resolution in a murine model of acute lung injury. Mucosal Immunology. 6 (2), 256-266 (2013).

10. Abdulnour, R.-E. E. et al. Phospholipase D isoforms differentially regulate leukocyte responses to acute lung injury. Journal of Leukocyte Biology. 103 (5), 919-932, (2018).

11. Fukunaga, K., Kohli, P., Bonnans, C., Fredenburgh, L. E., Levy, B. D. Cyclooxygenase 2 Plays a Pivotal Role in the Resolution of Acute Lung Injury. Journal of Immunology. 174 (8), 5033 LP-5039 (2005).

12. Colby, J. K. et al. Resolvin D3 and Aspirin-Triggered Resolvin D3 Are Protective for Injured Epithelia. American Journal of Pathology. 186 (7), 1801-1813 (2016).

13. Bonnans, C., Fukunaga, K., Keledjian, R., Petasis, N. A., Levy, B. D. Regulation of phosphatidylinositol 3-kinase by polyisoprenyl phosphates in neutrophil-mediated tissue injury. The Journal of Experimental Medicine. 203 (4), 857-863 (2006).

14. Mandell, L. A., Niederman, M. S. Aspiration Pneumonia. New England Journal of Medicine. 380 (7), 651-663 (2019). 
15. Seki, $\mathrm{H}$. et al. The anti-inflammatory and proresolving mediator resolvin E1 protects mice from bacterial pneumonia and acute lung injury. Journal of Immunology. 184 (2), 836-843 (2010).

16. Abdulnour, R. E. et al. Aspirin-triggered resolvin D1 is produced during self-resolving gram-negative bacterial pneumonia and regulates host immune responses for the resolution of lung inflammation. Mucosal Immunology. 9 (5), 1278-1287 (2016).

17. Traber, K. E. et al. Myeloid-epithelial cross talk coordinates synthesis of the tissue-protective cytokine leukemia inhibitory factor during pneumonia. American journal of physiology. Lung Cellular and Molecular Physiology. 313 (3), L548-L558 (2017).

18. Yamamoto, K. et al. Roles of lung epithelium in neutrophil recruitment during pneumococcal pneumonia. American Journal of Respiratory Cell and Molecular Biology. 50 (2), 253-262 (2014).

19. Acute Respiratory Distress Syndrome Network. Ventilation with Lower Tidal Volumes as Compared with Traditional Tidal Volumes for Acute Lung Injury and the Acute Respiratory Distress Syndrome. New England Journal of Medicine. 342 (18), 1301-1308 (2000).

20. Peng, X. et al. Inducible nitric oxide synthase contributes to ventilator-induced lung injury. American Journal of Respiratory and Critical Care Medicine. 172 (4), 470-479 (2005)

21. Abdulnour, R.-E. E. et al. Mechanical stress activates xanthine oxidoreductase through MAP kinase-dependent pathways. American Journal of Physiology-Lung Cellular and Molecular Physiology. 291 (3), L345-L353 (2006).

22. Serhan, C. N., Levy, B. D. Resolvins in inflammation: emergence of the pro-resolving superfamily of mediators. The Journal of Clinical Investigation. 128 (7), 2657-2669 (2018). 